large column of cylindrical oedematous tissue extending up from the site of the major injury going up seven segments in the cord; he also found obliterations in the spinal artery system. These are not new findings because they are already mentioned in the wonderful paper by Buss and Greenfield in I9I9.

And lastly, Hans Frankel may be interested to know that we too also tried heparin and dindevan without any success.

Dr. Whatmore (Edinburgh). We see this problem fairly commonly in neurosurgery, particularly with spinal cord lesions, particularly tumours. Most of these cases occur postoperatively, even with gentle surgical manipulation, and we have no doubt at all in our minds that in many cases this is due to oedema and responds very well to drugs like decadron.

To give you an example quickly. One month ago Mr. Harris had a patient with syringomyelia. This man came to us with weakness in his lower limbs, and a sensory level at about T6. Three days after the operation his level ascended to about the TI level, and he developed a complete paraplegia. He was immediately placed on decadron, Io ml. intravenously and thereafter $4 \mathrm{ml}$. intramuscularly for six hours, and within two days his recovery was complete-that is, he came back to his original level. He went on to recover even further, and is now walking.

Dr. BEDBRook (Australia). Well, ladies and gentlemen, I think you will agree that this is a most interesting subject, and one for co-operation between us all with Dr. Frankel.

\title{
CARE OF THE HAND IN CERVICAL SPINAL CORD INJURIES
}

\author{
By Alvin A. Freehafer, M.D. \\ Division of Orthopaedic Surgery, Western Reserve University School of Medicine, Highland \\ View Hospital and Veterans Administrations Hospital, Cleveland, Ohio
}

SPINAL paralysis involving the upper extremity causes various degrees of functional loss. Trauma to the cervical spinal cord with paralysis often leads to certain patterns which are based on the presence of functioning key muscle groups. The muscle function which remains is the basis upon which proper treatment can be provided. The most important aspect of care in any paralytic situation is an accurate examination to grade the muscle strengths, list the functioning muscles, and test for sensation. This information must be recorded and repeated at intervals.

The number of patients who sustain and survive cervical spinal cord injury is increasing. A few years ago many of these patients died or became hopelessly incapacitated. With skilful management these patients now survive and are able to become useful citizens in society. It is our duty to provide the kind of care and assistance needed to achieve independence.

Proper care of the hand in cervical spinal cord injury represents a small part in the management of this kind of severely handicapped person. It also represents a small part of the upper extremity. Needless to say management of spinal paralysis is difficult and complex, requiring the help of many specialists. All aspects of the patient's mental and physical problems must be considered. Although the hand represents a small part of the patient it is extremely important, especially to the patient with severe paralysis. A systematic and organised approach to its management is essential if maximum rehabilitation is to be achieved. 
During the past eight years more than fifty-five cervical spinal cord injury patients have been under direct observation at Highland View Hospital. More than 200 have been seen on consultation. Experience with these patients serves as a basis for the observations and recommendations made in this communication.

\section{LEVELS OF INJURY}

Most injuries to the spinal cord cause fairly well-defined levels of neurological loss. This neurological loss and the remaining function serve as a basis for prescribing treatment, and predicting functional potential so that a well-formed plan can be instituted early and carried out to achieve a preconceived goal. Changes may be necessary from time to time but the most important determinant is the neurological function.

Not all spinal cord injury results in distinct levels of injury. Recovery of neurological functions of varying types and degrees occurs sometimes but the majority of spinal cord injury patients show levels of neurological deficit. It is only these for whom generalities can be made.

Garrett et al. (I964) have classified cervical cord injuries into four levels of disability:

I. loss of hand intrinsics;

2. loss of all hand musculature;

3. loss of triceps and latissimus;

4. loss of wrist extension and poor arm control.

The intrinsic muscles of the hand are weakened or lost with the lowest level of cervical cord injury. The rest of the upper extremity is usually normal so that a patient with this loss can be expected to become independent at a wheelchair level in transfers, activities of daily living, bowel and bladder care, and automobile driving. This type of disability should not prevent the average patient from attending school or working outside of the home. Disability of hand function in this group is based on decreased ability to oppose the thumb to the other fingers and finger extension and flexion. Complete loss of hand intrinsics will make grasp less accurate and effective. Varying degrees of weakness may provide hand function approaching normal.

At a slightly higher level all voluntary muscle function in the hand is lost. Remaining muscle function of the wrist and forearm usually includes:

Extensor carpi radialis longus;

Extensor carpi radialis brevis;

Flexor carpi radialis;

Pronator teres;

Brachioradialis.

Grasp in this situation is much less effective and depends on the patient's ability to extend the wrist while tension of the volar structures produces finger flexion. This automatic grasp is weak and lacks dexterity. It is enhanced when spasticity of finger flexors occurs with wrist extension. This group of patients can be almost as independent as the first group but with increased difficulty and effort. Bowel and bladder care often requires assistance. For many activities the wrist driven hinge splint and self-help aids are necessary. 
In a slightly higher cervical cord injury, the triceps and latissimus dorsi are absent. The only voluntary muscle function in the wrist and forearm are:

Extensor carpi radialis longus;

Extensor carpi radialis brevis;

Brachioradialis.

The functional potential is similar to the second group but activities are slightly more difficult. Many of these patients learn transfers, activities of daily living, and automobile driving. A few learn bowel and bladder care but assistance is usually necessary. The wrist driven hinge splint and self-help aids are very valuable and provide independence in many activities. Many of these patients attend school and work outside of the home.

At the highest cord level usually compatible with life no voluntary function exists below the elbow. Biceps and shoulder function are weak or absent. External power is needed if prehension is to be obtained. The hinge splint with external power can be used successfully. Initiation of function can be made by head movement, shoulder elevation, or arm motions. If elbow and shoulder function is too weak a ball-bearing feeder or the 'Rancho Mobile Arm' is helpful. These patients are severely handicapped but much can be done to make them happier and more useful in society.

The classification of levels is of course an arbitrary one for purposes of discussion. While most patients fall into one of the groupings, situations exist where there is overlap. For example, it is not infrequent to see finger extensors present with the second group. Occasionally the radial wrist extensors and brachioradialis are present but wrist extension is too weak to produce automatic closure of the fingers. Neurological levels, while usually symmetrical, may sometimes be different in the two upper limbs. Sometimes very high levels of cord injury leave no upper limb function but spare the phrenic nerves. Some patients maintain partial neurological function below the cord injury.

Most patients do, however, sustain rather distinct levels of neurological loss as a result of cord injury. Understanding of these patients serves as a basis for study and treatment planning.

\section{EARLY CARE}

Immediately after the onset of spinal cord injury and paralysis, treatment of course is directed toward maintaining life. Reduction of the fracture dislocation follows as soon as possible and is usually done with skull traction with the patient on a turning frame. Replacement of the malaligned cervical spine must be done early and with care. When alignment of the cervical spine has been accomplished and the size of the spinal canal has been restored, immobilisation must be accomplished by maintenance of recumbency on a frame, bracing, or fusion. Laminectomy is sometimes indicated but should be carried out after cervical canal alignment has been restored.

This early management is very important and is mentioned very briefly only to indicate that the spinal cord injury patient is going to be recumbent for several weeks or months depending on the nature of injury and the success achieved from reduction and neck stability. Care of the skin, urinary tract, respiratory system, musculoskeletal system should be begun with the admission of the patient. It is 
at the beginning of this period of recumbency that care of the hand must start. The proper care includes: (a) examination of muscle strength and sensation; $(b)$ proper positioning of hands; $(c)$ range of motion of joints.

The motor power of muscles should be graded and recorded along with results of sensory examination. The results of neurological examination should be charted on admission and repeated at regular intervals. From this an accurate assessment of subsequent functional capacity can be made. There are often considerable changes in neurological function in the first eight weeks after injury. Because root damage is frequent neurological changes often occur for a period of up to a year.

Positioning of the hand serves several purposes. Prevention of pressure areas, maintanence of the wrist and fingers in a functional position so that if stiffness does occur the hand will not be useless, and the proper posturing to eventually allow maximum function. A hand placed flat on the armrest of the turning frame will surely develop into a stiff flat hand. The hand which is permitted to hang unsupported will often result in a stretch palsy of the wrist extensors. A roll of webril or wheet wadding about one to two inches in diameter placed so that the thumb opposes the fingers is a simple and effective way to maintain proper hand posture while waiting for more sophisticated braces.

Range of motion of the upper limb should be done by physical therapist, occupational therapist, and nurse. Relatives should be instructed so they can do it as well. This gives the patient several treatments each day. Particular attention should be given to passive shoulder abduction, elbow extension, metacarpophalangeal flexion and thumb abduction but not to the exclusion of other motions. When appropriate, active muscle function should be performed and should lead to active resistance exercises. This has great application with regard to wrist extension. The automatic hand with its tenodesis effect is desired and efforts should be directed to making it as effective as possible.

\section{LATE CARE}

At six to eight weeks following injury and shortly thereafter most spinal cord injury patients are up in a chair or in the process of getting to the chair. This is a period when the patient may be getting some insight into his problem and when the spinal cord injury service is making a maximum effort to educate the patient on the many new ways of living he must learn. This education includes training in the use of the hand.

It is desirable to have a normal hand functioning in all its customary activities that are expected in the normal person, but the spinal cord injury patient with paralysis uses his hands differently. This may not always be desirable but with paralysis he has no choice. For example, the tenodesis effect in the paralysed hand is present in all persons but becomes more pronounced in spinal cord injury patients when weakness becomes more severe.

Paralysis of the trunk and lower limbs requires that the patient use his hands differently. He must do wheelchair push-ups, and transfer from chair, to bed, to car, etc., using his hands and upper limbs. In order to transfer the hand must be flat enough to give support on a flat surface. The quadriplegic often locks his wrist in dorsiflexion to grab a trapeze or to move his legs. He sometimes uses his 
arms much like a tightrope walker to maintain balance. He often uses his hands as clubs to maintain balance or to stop leg spasms.

It must also be realised that the paralysed hand must accomplish for the patient the same things that the normal hand does. Most quadriplegics have two wrist extensors which operate a grasping device. This grasp is poor compared to active voluntary finger closure and opposition. Certain factors, however, make the automatic grasp very efficient. Some spasticity and tightness of the finger

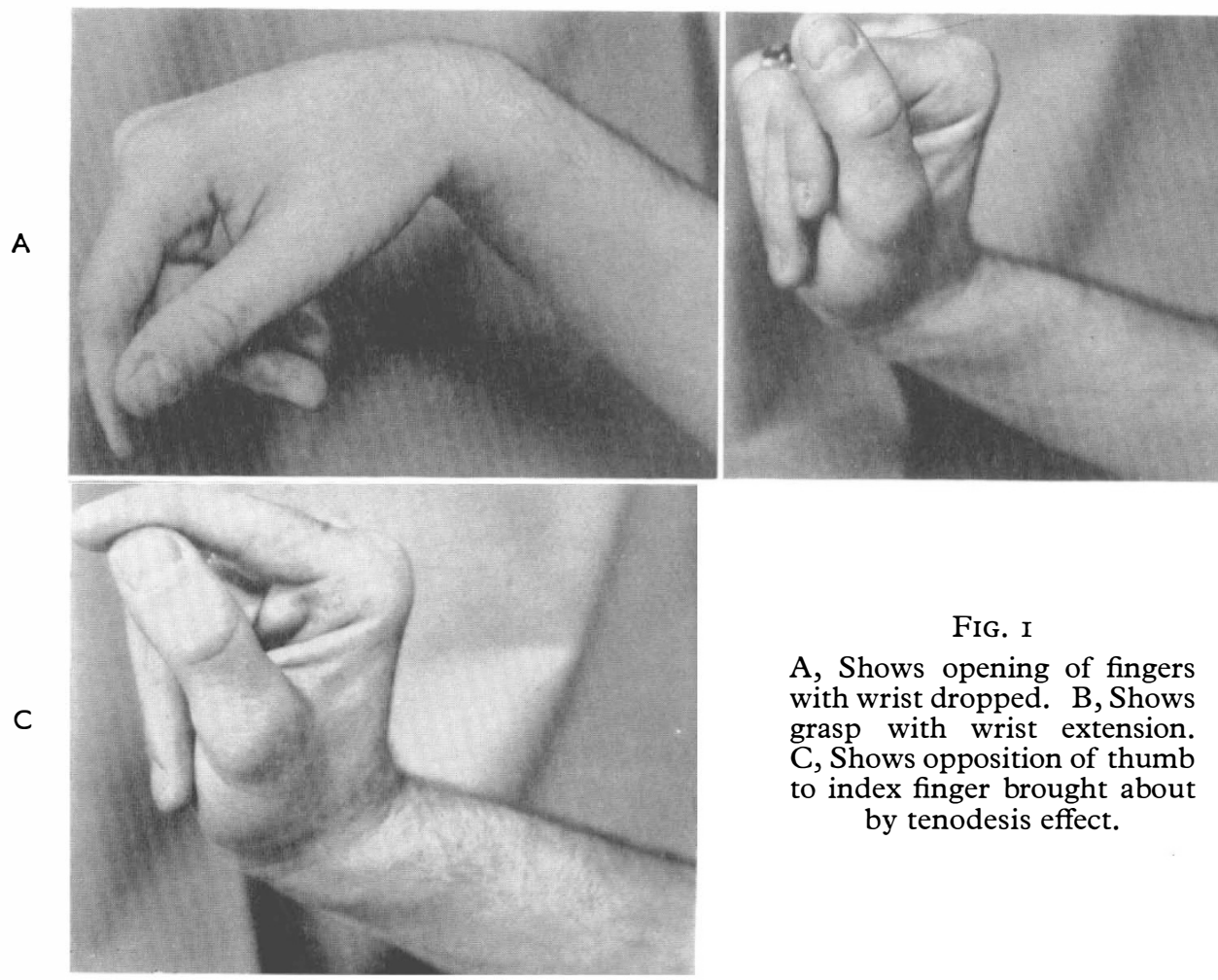

flexors is desirable in increasing strength. At the same time a degree of mobility of the palm, thumb, and metacarpo-phalangeal joints is desirable. Figure I, A, $\mathrm{B}$, and $\mathrm{C}$ show an excellent functional hand. Only voluntary wrist extension motors this hand but because of a combination of mobility, tightness, spasticity of finger flexors, thumb flexors, and thumb adductors, and good finger position this patient is independent. He can button and unbutton clothes, feed himself, lift books, care for his external catheter system, manage his bowels and many other activities with no splints or self-help aids. He accomplishes all of these activities with his ability to passively close his fingers and oppose his thumb to the side of the index finger.

A great deal of organised effort is directed to maintaining a good posture to the hand and to strengthening wrist extension when present. This provides a hand which can be normal if neurological function returns and can be functional with or without external splints and aids if paralysis persists. 
The methods used to improve upper limb function are: (a) positioning and proper exercises; (b) external splints and aids; and (c) surgical procedures. Emphasis of methods used depends on the remaining neurological function.

Table I is a list of surgical procedures performed. Only a small percentage of spinal cord injury patients need reconstructive surgery of the hand. The indications are to correct disabling deformities and to improve function. With good preparation the deformities should not occur unless spasticity is uncontrollable.

\section{TABLE I}

Procedures Performed

Tenotomy
Transfer for opposition
Transfer for wrist extension
Transfer for finger flexion
Flexor tenodesis
Extensor tenodesis
Surgical wrist driven hand
Intrinsic release
Intrinsic transfer

It is not possible to restore severely paralysed hands to normal. Only when one or two functions are missing can one expect to restore the hand so that it approaches normal. Restoration of one or two functions to a paralysed hand, however, may provide much needed help. With good care of the hand the spinal cord injury patient rarely needs surgical correction. He uses his hands differently and any surgical assistance that can be made must not be thought as of restoring normalcy.

In general it seems prudent to consider surgical procedures after one year from injury and after a good conservative approach has failed to give desired or expected function. The patient must be interested and want help. He should be rehabilitated maximally based on his neurological examination.

When indicated opponens transfer, transfer to improve finger flexion and wrist extension are very helpful. Tenodesis (Wilson, 1956) of finger flexion or extension is helpful and gives strength but results often leave much to be desired when compared to voluntary power. Arthrodesis should not be done at the wrist but fusions of the interphalangeal joints may be a good approach to deformed fingers. The surgical wrist driven hand (Nickel et al., I963) is difficult to construct and results in my experience have been poor.

Surgery done on spinal cord injury patients is not easy. Respiratory function, urinary tract problems, skin care are only a few reasons why hand surgery should be well planned. Healing of tendon transfers is delayed when compared to the normal person. Retraining of tendon transfers is difficult and prolonged. The end result of the surgeon's efforts is often less dramatic when compared to less severely paralysed patients. With the proper approach to the problem and a knowledge of spinal cord injury, reconstructive surgery of the hand can be very helpful to the disabled patient.

In the first level of disability where intrinsic muscles have been lost or weakened the function of the hand can be made almost normal by opponens transfer. This depends on absence of hand deformity and on sufficient muscle strength. One year should be allowed for regeneration of nerve root damage to occur, however. 
During this time the patient is busy enough learning to adjust to his disability anyway. Efforts are directed to muscle strengthening and use of the short or long opponens splint. Figure $2 \mathrm{~A}$ shows one hand to have poor opposition. Figure 2B shows improvement following opponens transfer done by a modification of Royle's method (Royle, I938; Thompson, I942).

The group 2 disability is characterised by the presence of the following actively functioning forearm muscles:

Extensor carpi radialis longus;

Extensor carpi radialis brevis;

Flexor carpi radialis;

Pronator teres;

Brachioradialis.

Patients with this kind of disability should be taught to use the hand in automatic fashion to take advantage of the tenodesis effect of the finger and thumb flexors.

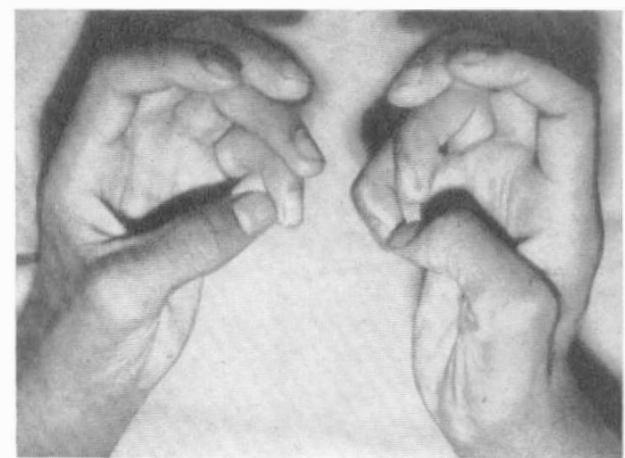

A

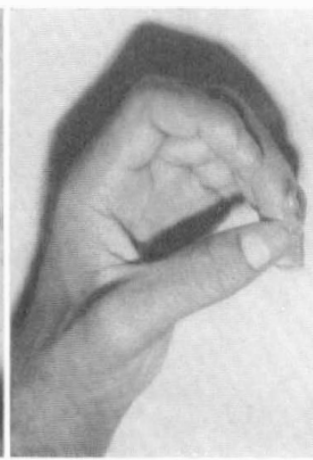

B

FIG. 2

A, Shows poor thumb opposition on right.

B, Shows good thumb opposition following transfer.

The posturing of the hand is important. The wrist driven hinge splint is very useful for this group of patients. The patient should be taught how to apply and detach it, and to use it for specific tasks which cannot be performed effectively otherwise. Most patients will eventually use the hinge splints for a few specific tasks such as applying external catheter drainage or picking up small objects or feeding. Others will discard them and use automatic grasp or self-help aids. The great value of the hinge splint, however, is that fine and intricate tasks may be performed which otherwise might not be accomplished. It also is a good training device to maintain the fingers and thumb in good position and in improving wrist extensor function for automatic grasp.

Surgical reconstruction is sometimes valuable in this group of patients. The majority do not need it, especially if they have good grasp with wrist extension or have mastered the use of the wrist driven hinge splint. Those who have poor grasp or who would benefit from some strength in one hand can be helped. Figure 3A shows the hand of a patient who had no ability to grasp. She also had hypermobility in extension at the metacarpo-phalangeal joints. The volar capsules were shortened by Zancolli's method (I942), the flexor carpi radialis was used to 


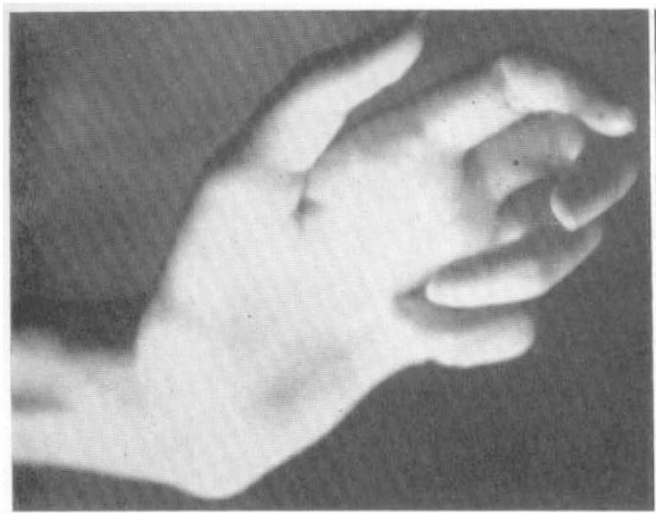

A

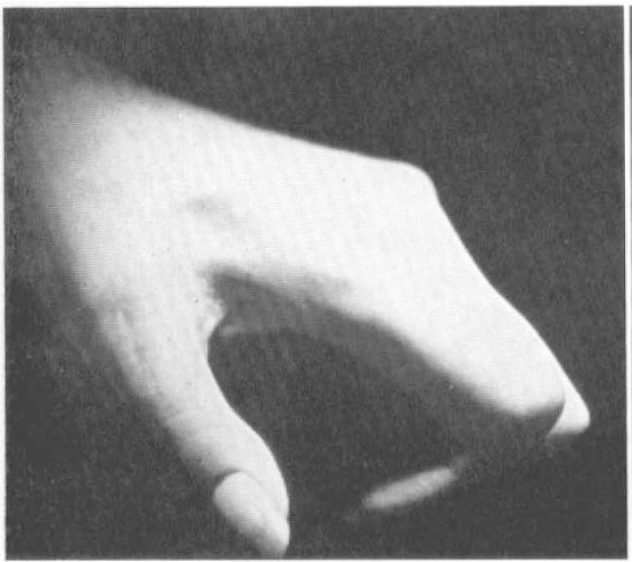

C

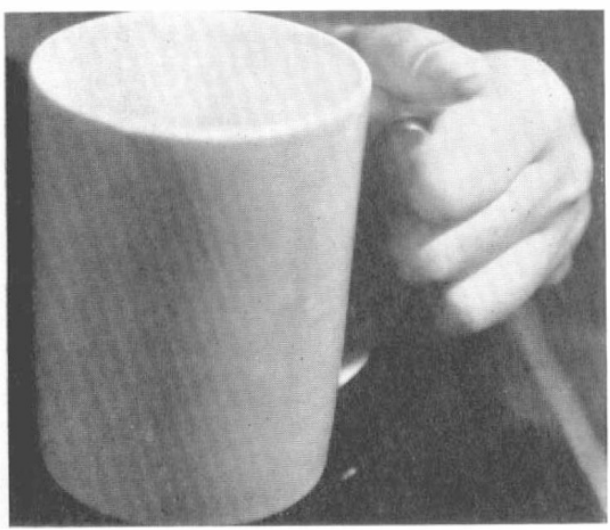

E

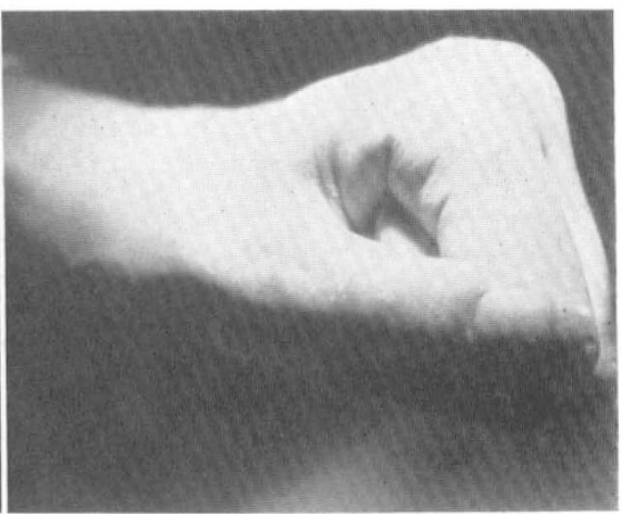

B

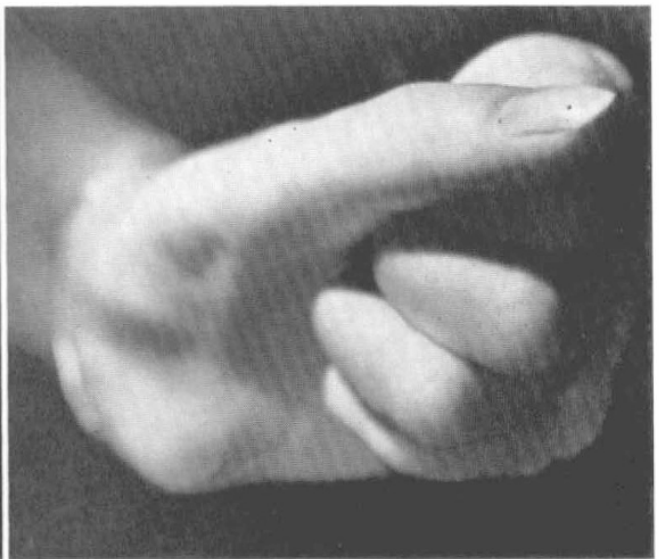

D

FIG. 3

A, Shows no effective grasp on wrist extension. B, Shows grasp after transfers to improve finger flexion and thumb opposition. C, Shows finger opening with wrist dropped. D, Shows opponens transfer across the palm. E, Shows patient lifting cup. 
motor the flexor digitorum profundus, and the extensor carpi radialis longus was brought around the ulnar side of the wrist and attached to the extensor pollicis brevis which was rerouted across the volar aspect of the palm. Figure 3, B, C, D and $\mathrm{E}$ show the result. She has good finger flexor power and side to side thumb

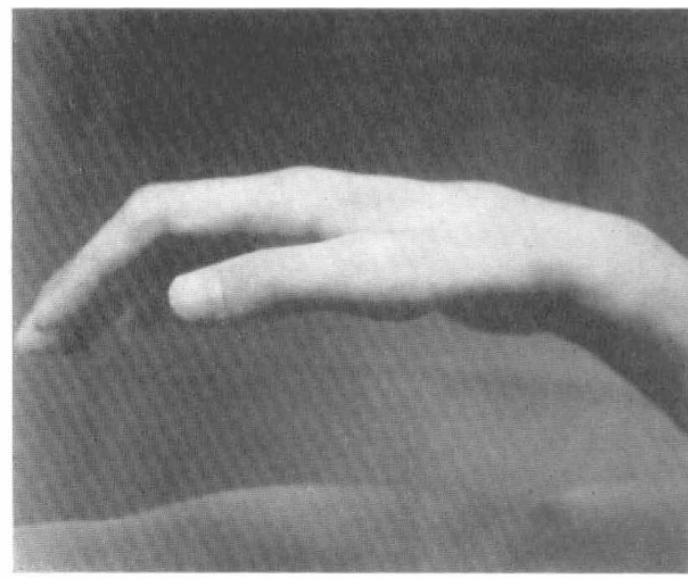

A

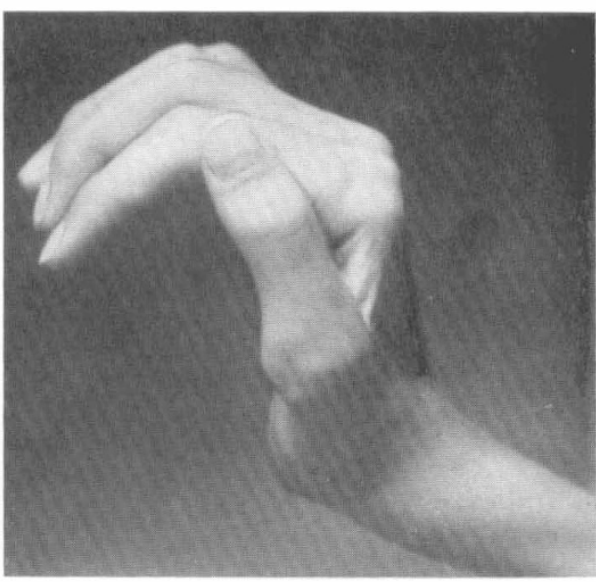

B

FIG. 4

A, Shows opening of fingers with wrist dropped. B, Shows finger closure with wrist extension.

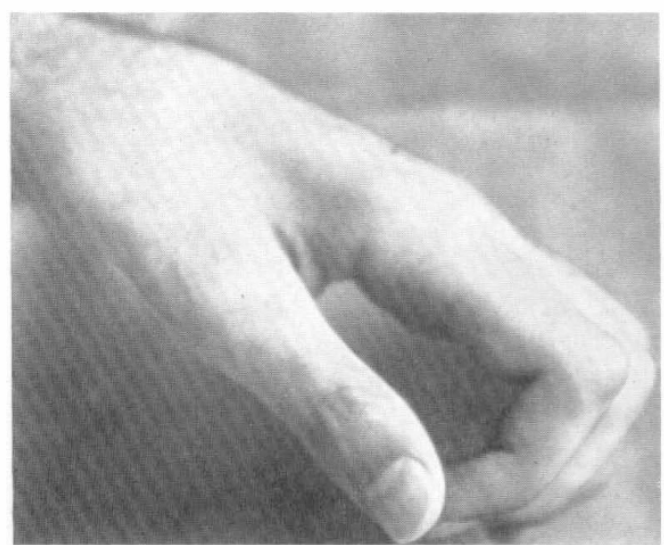

C

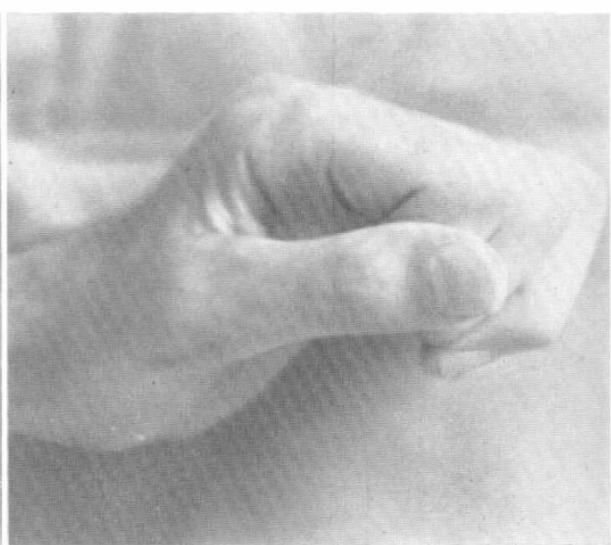

$\mathrm{D}$

FIG. 4

C, Shows finger opening with wrist dropped. D, Shows finger closure with wrist extension.

pinch which are enhanced by extension of the wrist. Because there is a muscle imbalance she has developed flexion deformities of the proximal interphalangeal joints. Function, however, is much improved. Perhaps a better result could have been obtained by the method described by Lipscomb et al. (1958), which requires two steps but gives finger flexion and extension and opposition.

With group 3 disabilities only the two radial wrist extensors and the brachio- 


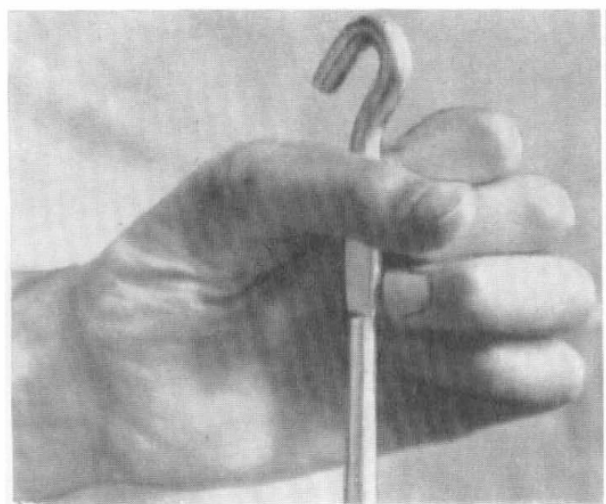

$\mathrm{E}$

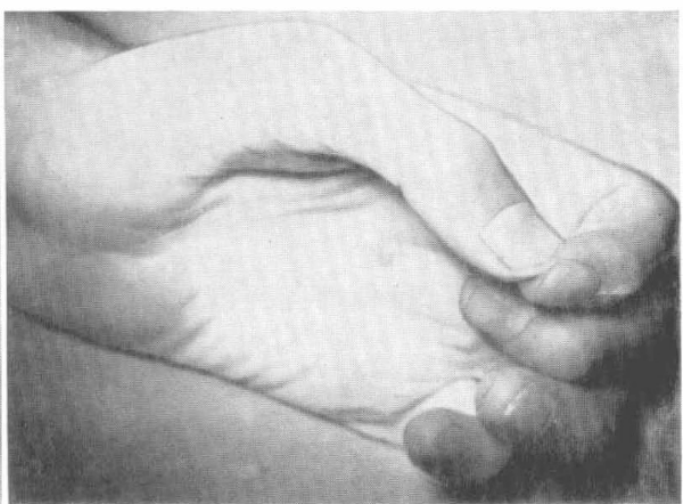

$\mathrm{F}$

FIG. 4

E, Shows patient lifting two-pound weight. F, Shows opponens transfer across palm.

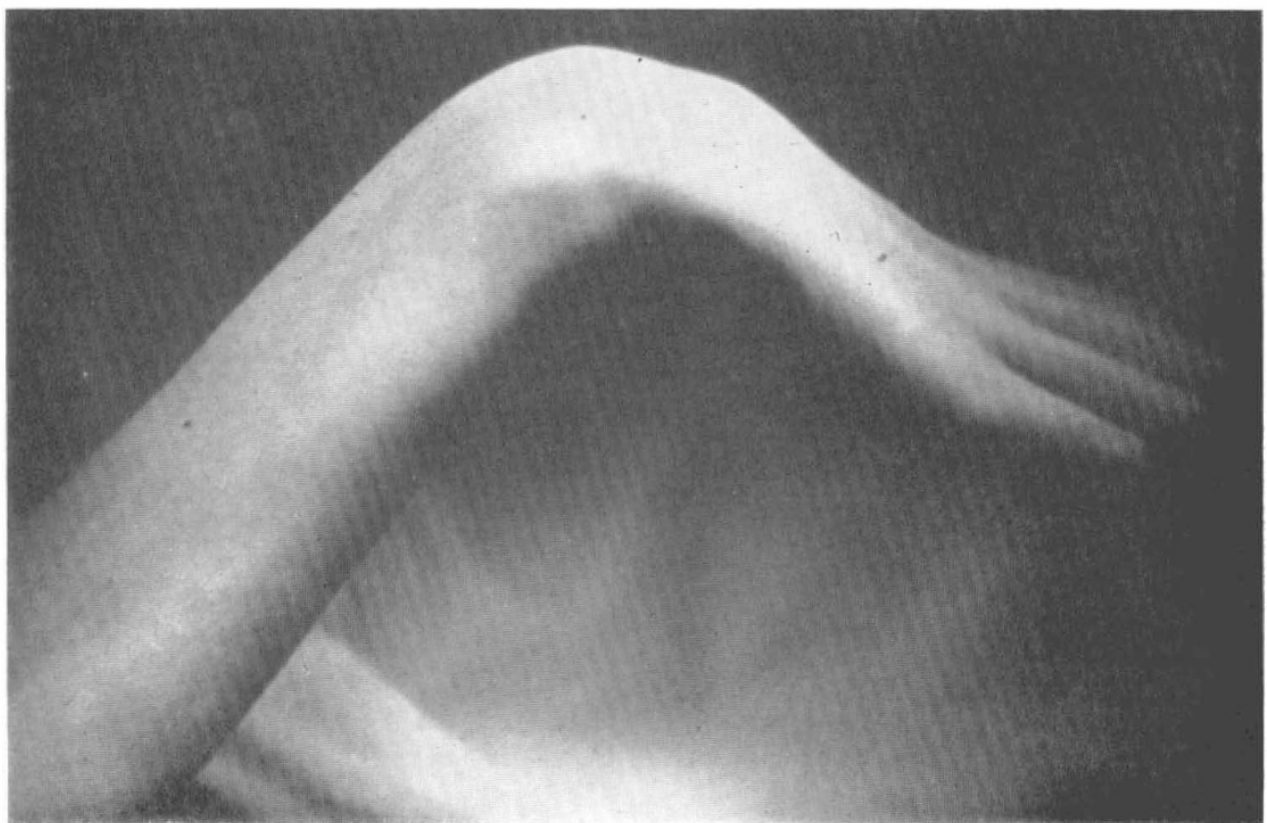

A

FIG. 5

A, Shows paralysis of wrist extension. 
radialis are present in the forearm. The approach to treatment is the same as in group 2. A small percentage of patients who have poor finger closure or want strength can be benefited by using muscles to motor finger flexion and opposition. The extensor carpi radialis longus can be used for finger flexion and the brachioradialis for opposition. It is important to use the long wrist extensor for transfer otherwise radial deviation will occur with wrist extension. Active wrist extension must not be lost because it represents the most important muscle function. Because a muscle imbalance is created flexion deformity develops at the proximal interphalangeal joints. Figure 4, A and B show a hand with no grasp and which is functionally poor. After transfer finger grasp and side to side pinch are good (fig. 4, C, D, E and F ).

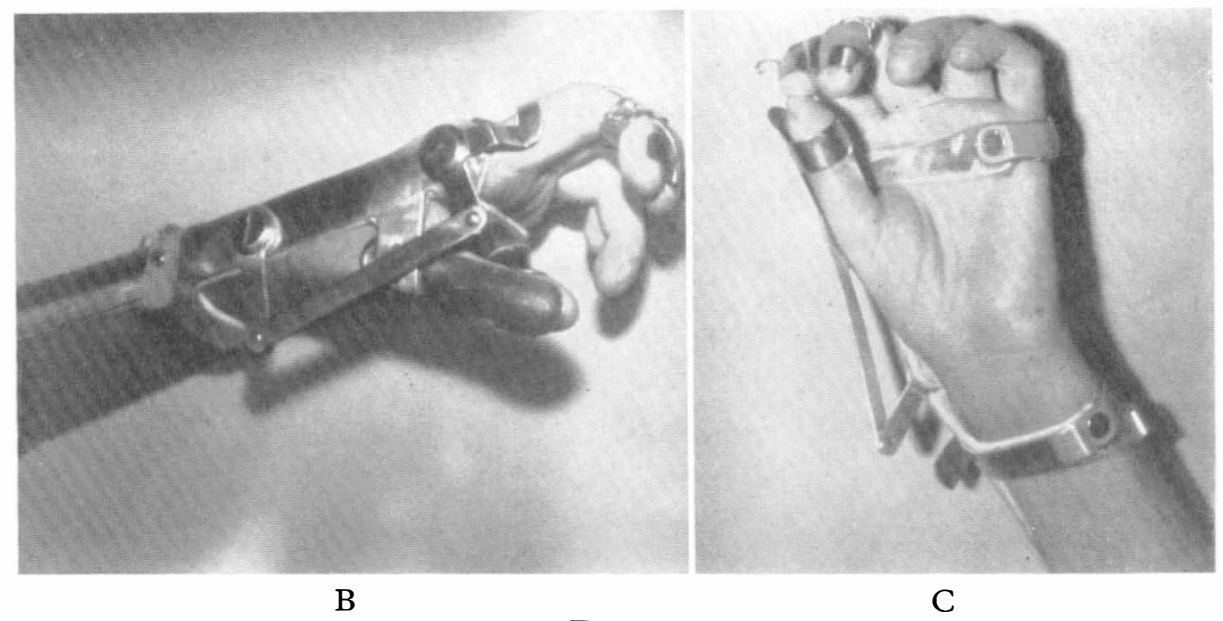

FIG. 5

B, Shows finger opening wrist of wrist driven hinge splint. C, Shows finger closure with wrist driven hinge splint.

Between group 3 and group 4 disabilities an occasional situation exists where wrist extension is weak or absent with the brachioradialis functioning (Freehafer and Mast, 1967). Figure 5A shows a wrist with no wrist extension. Figure 5, B and $\mathrm{C}$ show how function is markedly improved with transfer of the brachioradialis into both radial wrist extensors or into the short radial wrist extensor.

Group 4 disabilities require external power if functional activity of the hand is to occur. Hand posture and proper exercising is important so that external devices can be used. The hinge splint is usually used to provide grasp and is motored by $\mathrm{CO}_{2}$ attached to a McKibben muscle, electric motors or myoelectric control. Much work is being done in the field of external power and more remains to be accomplished. $\mathrm{CO}_{2}$ and electric motors are the most practical at this stage.

Many of the patients in this group are unable to control their shoulders and elbows effectively. They will require a feeder arm or one of its modifications.

\section{SUMMARY}

The care of the hand in cervical spinal cord injury should be a well-organised programme in order to provide maximum function to the patient who is already 
severely disabled. The methods used should include careful examination and evaluation, attention to posture of the hand, range of motion exercises, the use of a wide variety of external aids, and occasionally judicious use of surgical techniques.

The rationale for such a programme is that such care will lead to a normal hand if neurological recovery occurs or the hand will be functional with or without splints and aids if paralysis persists.

It must be realised that the paralysed hand in permanent spinal cord injury will never be normal and that the patient will wisely take advantage of the tenodesis effect of the finger flexors as the wrist extends. Because most cord injury patients develop this automatic type hand, efforts should be made from the time of injury so that the hand and wrist will be functional. Efforts should develop the kind of position, mobility of the wrist and palm, and tightness of finger flexors to permit good closure of the fingers on wrist extension to allow functional use for wheelchair existence.

Surgery to the hand is valuable for a small percentage of patients. In general it is unwise to perform reconstructive procedures until enough time has been allowed for nerve root injuries to recover which is about one year following injury.

\section{REFERENCES}

FREEHAFER, A. A. \& MAST, W. A. (I967). Transfer of the Brachioradialis to Improve Wrist Extension in High Spinal Cord Injury. F. Bone and foint Surg. 49-A, 648.

Garrett, A. L., Perry, J. \& Nickel, V. L. (I964). Traumatic Quadriplegia. F.A.M.A. I 87, 7 .

Lipscomb, P. R., Elkins, E. C. \& Henderson, E. D. (1958). Tendon Transfers to Restore Function of Hands in Tetraplegia, Especially after Fracture Dislocation of the Sixth Cervical Vertebra on the Seventh. F. Bone and foint Surg. 40-A, I07I.

Nickel, V. L., Perry, J. \& Garrett, A. L. (1963). Development of Useful Function in the Severely Paralyzed Hand. F. Bone and foint Surg. 45-A, 933.

Royle, N. D. (1938). An Operation for Paralysis of the Intrinsic Muscles of the Thumb. F.A.M.A. i, $6 \mathrm{I} 2$.

Thompson, T. C. (I942). Modified Operation for Opponens Paralysis. F. Bone and foint Surg. $24,632$.

WiLson, J. N. (1956). Providing Automatic Grasp by Flexor Tenodesis. F. Bone and foint Surg. 38-A, IOI9.

ZANColi, E. A. (I942). Claw-hand caused by Paralysis of the Intrinsic Muscles. A Simple Surgical Procedure for its Correction. F. Bone and foint Surg. 24, 632.

\section{Discussion}

Dr. McSweEnEy (Great Britain). I just want to make three small points. One, I am in full agreement, or almost full agreement, with Dr. Freehafer when he says that only Io per cent. of his cases-this is applicable to the hand only, I am not considering the upper limb transferences-only Io per cent. require surgery. This, in fact, is 50 per cent. more than I would do.

The second point is to wait at least twelve months. Again, I would exceed this by three or four months. And the last point is a point that has been emphasised a few times already in other connections, that no two cases are exactly alike. In other words, I think it is an error to say that 'this man has a $\mathrm{C}_{7}$ lesion, a C6 lesion, ergo he must have such and such a transfer'. And this, I think, has been the fault in previous papers published in other journals. It has assumed an anatomical exactitude which doesn't exist. 
Dr. BEDBRook (Australia). I would just like to make one point on this paper myself, and that is that I would agree with both the previous speakers, particularly Mr. McSweeney, that only about 5 per cent. of the material I see comes to anything in the way of surgical reconstruction. And then I have one very specific rule, that I will carry out no reconstructive surgery on cases with any spasticity.

Many years ago, when I was starting this work, I did this, and finished up with a couple of appalling hands. I have decided that this was one rule I would never break, and I have not broken it since. I would be very interested to know if anybody has operated on these spastic cases. I think that this reconstructive work we have been talking about has a very small place in a very small group of patients, but it possibly does help the odd patient from time to time, and then only in the flaccid cases. I welcome any further discussion.

Sir Ludwig GutTmann (Great Britain). I can only endorse what the three speakers have said from my own experience. As Dr. Freehafer quite rightly said, we can, with a. lesion below C6, readjust the function of the arm and hand to such a degree that these people can do a lot of things in their daily activities, including dressing and buttoning-up. Of course, in these cases we have, already in the early stages of our work, encouraged the people: 'If you can't use one hand, use both hands'. Once the tetraplegic knows the compensatory facilities he has in his remaining muscles in the arm, he will mobilise them, and this includes also activity in sport. To-day tetraplegics are able to take part in table-tennis. Many people ask me how it is possible that a man without fingers can play table-tennis. As long as he has the extensor carpi-radialis working, you fix the table-tennis bat on his hand with a bandage, and he plays with his wrist, elbow and his arm.

I entirely agree that in cases with spasticity one can do endless harm, and I have unfortunately seen patients, not only with spasticity, who had a transplantation of the wrist flexor on the extensor digitorum longus. The result was a claw-hand, and the patient was extremely unhappy about it. I remember one particular case, a civil servant, who came with profound contractures of all fingers in flexion and Dr. Michaelis had to undo what the transplanting surgery had done to harm the man.

I would like to add two important points. One is the prevention of contractures in the early treatment of the hands as well as the elbows. People with a C6 lesion come in with profound flexion contractures of the forearms as a result of the paralysis of the triceps and the uninhibited action of the biceps. Once the contractures develop in the elbow, we have increased the tetraplegic disability by our bad treatment. In the triceps lesion this is particularly important because the triceps is a plurisegmental muscle C6, $\mathrm{C}_{7}$, C8. As Bedbrook and McSweeney quite rightly pointed out, every case has to be considered individually and this applies to the segmental supply of the triceps. For instance, in some cases the supply of C6 can be so strong that this only segment can compensate later the two other segments which are knocked out. In other words, if the patient cannot at first extend his forearm, he will learn it later, when this one segment takes over its compensatory innervation. But if the triceps, in the acute stages, is overstretched, by faulty position of the forearm in flexion, of course, then even if the C6 segment recovers that muscle will never be functionally good.

The second point. C8 lesions are, of course, mobile in their wheelchairs. But if you see them after a certain time, the area of their palm is black and extremely damaged by continuous pressure when driving their wheelchair. Now in these cases one should train the physiotherapist and the nursing staff to watch the care of the palm of the hand. Just two days ago I saw two sportsmen, C8, with dreadful hands, which endangers, in due course, not only the function, but if they get an infection the remaining muscles are also damaged. 